\title{
Chromospheric activity of late-type stars based on Guoshoujing Telescope
}

\author{
Liyun Zhang ${ }^{1}$, Jianrong $\mathrm{Shi}^{2}$, Jingkun Zhao $^{2}$, Ali Luo ${ }^{2}$, \\ Guoyin Zhang ${ }^{1}$, Qingfeng $\mathrm{Pi}^{1}$ and for the Guoshoujing \\ Telescope Collaboration \\ ${ }^{1}$ College of Science/Department of Physics, Guizhou University, Guiyang 550025, China \\ email: Liy_zhang@hotmail.com \\ ${ }^{2}$ National Astronomical Observatories, Chinese Academy of Sciences, Beijing 100012, China
}

\begin{abstract}
We introduced our preliminary results of chromospheric activity of late-type stars based on the stellar spectrum of the pilot survey of the Large Sky Area Multi-object Fiber Spectroscopic Telescope (LAMOST, also called Guo Shou Jing telescope). We have found 1151 active $\mathrm{M}$ stars from $17471 \mathrm{M}$ samples using the chromospheric active indicator of the $\mathrm{H}_{\alpha}$ line.
\end{abstract}

Keywords. stars: late-type, stars: activity, stars: chromospheres, stars: flare, stars: low-mass.

\section{Introduction}

Late-type stars with thick convective zones and rapid rotation exhibit magnetic activity phenomena, such as plage and flare in the chromosphere. Chromospheric activity produce fill-in or emission in the chromospheric active indicators of the Ca II H \& K, He I D3, $\mathrm{Na}$ I D1 D2, Mg I b triplet lines, $\mathrm{H}_{\alpha}, \mathrm{H}_{\beta}$ and other Balmer lines, and Ca II IRT lines (Montes 2004; Zhang 2011; etc). We intend to research the common properties of the chromospheric activity on the basis of a large samples of late-type stars with different stellar parameters available from the survey of the Guoshoujing telescope. In the end, we want to obtain the precise relations of age-chromospheric activity-stellar parameters (such as rotational velocity, spectral type, chemical abundances, ...).

\section{Chromospheric activity based on Guo Shou Jing telescope}

Guo Shou Jing Telescope (Cui et al. 2012; Zhao et al. 2012) provides a wonderful chance to study chromospheric activity of late type stars (Zhang 2011). We have selected K7 and M stars (V2.3.3) from the LAMOST pilot survey (Luo et al. 2012) in the disk (Chen et al. 2012) and anti-center of the Milky Way (Deng et al. 2012) in Oct. Nov. Dec 2011, and Mar. 2012. Then, we visually inspected all candidates and manually assigned spectral types using the Hammer program (Hawley et al. 2002; West et al. 2004). Meanwhile, the chromospheric equivalent width of the $\mathrm{H}_{\alpha}$ line was measured using the Hammer program, which was made by integrating over the specific line region $\left(8 \AA\right.$ wide centered on $\left.\mathrm{H}_{\alpha}\right)$ and subtracting off the mean flux calculated from two adjacent continuum regions. The criteria similar to one of West et al. (2011) is used to classify the activity. We have found 1151 active M stars from 17471 samples. Spectra for active M 0-7 samples are displayed in Fig. 1. Fig. 2 (left) shows the fraction of stars that are active as a function of spectral type for LAMOST, and LAMOST+SDSS data. The results are consistent with result for M0-M6 stars (West et al. 2011). Two of them are also observed with $2.16 \mathrm{~m}$ telescope at the Xinglong station of the National Astronomical Observatories of China (Fig. 2 right). 


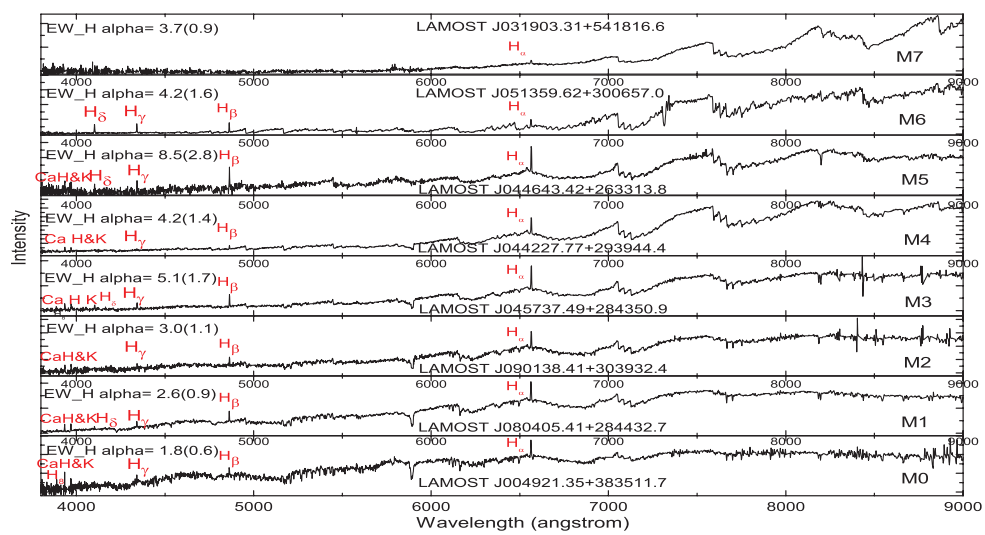

Figure 1. Spectra of the active M0-M7 stars from the LAMOST pilot survey.
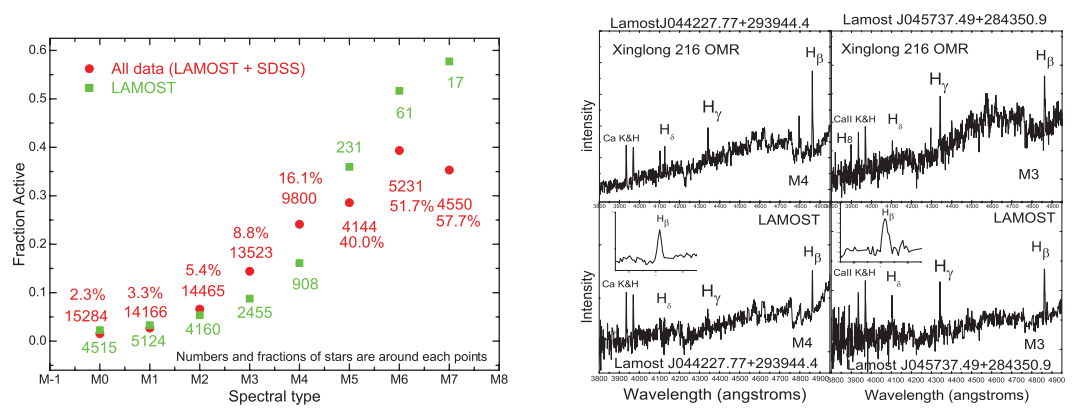

Figure 2. The fraction of active with spectral type for LAMOST (see online supplementary material Table $1 \& 2$ ), and LAMOST+SDSS data (left); Two active spectra using $2.16 \mathrm{~m}$ telescope and LAMOST, NAOC (right).

\section{Acknowledgements}

We are very grateful to Dr. Montes D., West A. A., Gu S. H., Han J. 1., and Zhou A. Y. Guoshoujing Telescope (the Large Sky Area Multi-Object Fiber Spectroscopic Telescope LAMOST) is a National Major Scientific Project built by the Chinese Academy of Sciences. The work is supported by the NSFC under grant No. 10978010 and 11263001.

\section{Supplementary materials and methods}

The Supplementary material referred to in this article can be found at http://dx.doi.org/10.1017/S174392131300255X

\section{References}

Chen, L. Hou, J. L., Yu, J. C., et al., 2012, RAA, 12, 805

Cui, X. Q., Zhao, Y. H., Chu, Y. Q., et al., 2012, RAA, 12, 1197

Deng, L. C., Newberg, H. J., Liu, C., et al., 2012, RAA, 12, 735

Hawley, S. L., Covey, K. R., Knapp, G. R., et al., 2002, AJ, 123, 3409

Luo, A. L., Zhang, H. T., Zhao, Y. H., Zhao, G., et al., 2012, RAA, 12, 1243

Montes, D., Crespo-chacón, I., Gálvez, M. C. et al., 2004, LNEA, 1, 119

West, A. A., Hawley, S. L., Walkowicz, L. M., et al., 2004, AJ, 128, 426

West, A. A., Morgan, D. P., Bochanski, J. J., et al., 2011, AJ, 141, 97

Zhang, L. Y., 2011, ASPC, 451,123

Zhao, G., Zhao, Y. H., Chu, Y. Q., et al., 2012, RAA, 12, 723 